\title{
Directional Analysis of 2D Cardiac Motion Slices Using the Discrete Helmholtz Hodge Decomposition
}

\author{
John A Sims ${ }^{1}$, Anderson Gabriel Santiago ${ }^{1}$, João L Salinet ${ }^{1}$, Marco Antonio Gutierrez ${ }^{2}$ \\ ${ }^{1}$ Biomedical Engineering, Engineering Modelling and Applied Social Sciences Center, Federal \\ University in ABC (UFABC), São Paulo, Brazil \\ ${ }^{2}$ Informatics Division, Heart Institute, University of São Paulo Medical School, São Paulo, Brazil
}

\begin{abstract}
The 2D Discrete Helmholtz Hodge Decomposition $(D H H D)$ is a candidate for decomposition of cardiac motion vector field (MVFs)from modalities such as echocardiography, allowing analyses of rotational, radial and harmonic MVFs separately and the tracking of centers of flow phenomena. However, the literature contains differing opinions about how boundary conditions (BCs) should be applied. In the proposed study, we investigate two sets of $B C s$, found in the literature, using synthetic motion vector fields: (i) zero BCs for all nodes along the domain boundary; (ii) a single zero BC for the first node. We found that (i) produces a decomposition with Normalised Root Mean Square Error (NRMSE) below 1\%, while (ii) produces NRMSE greater than $200 \%$ when the input field contains a harmonic component. Further, we demonstrate the decomposition of a synthetic motion field from the Extended Cardiac Torso Phantom v2 into radial, rotational and harmonic components.
\end{abstract}

\section{Introduction}

Quantification of the left ventricular (LV) motion field from a sequence of images along the cardiac cycle can provide valuable information about health and assist in diagnosis of diseases. Buckberg (2004), according to Notomi et al. [1], notes that research in clinical cardiac mechanics has moved towards three-dimensional ventricular deformation studies, which include investigations of LV torsion. We have previously applied the Discrete Helmholtz Hodge Decomposition (DHHD) to 3D cardiac motion fields, calculated from in-vivo 4D image sequences using optical flow techniques ([2]), and show that the DHHD algorithm presents the following possibilities for analysis: (i) an automated method for decomposing the motion field into components, such as LV torsion, which can then be studied independently and in a simpler manner; (ii) a method for tracking the centres of flow phenomena from frame to frame.

Polthier and Preuss [3] introduce the DHHD, showing how a smooth $2 \mathrm{D}$ vector field, $\vec{\xi}$, can be written in terms of the sum of a curl-free (CF) scalar potential, $D$, the curl of a divergence-free (DF) vector potential, $\vec{R}$, and a harmonic remainder, $\overrightarrow{F_{H}}$, which is both $\mathrm{CF}$ and $\mathrm{DF}$, and represents the linear flow (Equation 1).

$$
\vec{\xi}=\nabla D+\nabla \times \vec{R}+\overrightarrow{F_{H}}
$$

The DHHD requires the application of boundary conditions (BCs) to achieve a unique solution, and the consistency of BCs influences the orthogonality and uniqueness of the decomposition [4]. Guo et al. [5] apply BCs (hereafter referred to as Guo $\mathrm{BCs}$ ) to their $2 \mathrm{D}$ decomposition by constraining the first components of $D$ and $\vec{R}$ to zero, claiming that setting potentials to zero at only one node is sufficient for a unique decomposition. While no claims are made about the orthogonality of the decomposition, a recursive decomposition of the harmonic component is proposed to provide increased accuracy. Further, the need for this recursive solution is unclear, since the application of consistent BCs should result in a unique solution up to numerical precision [4]. Tong et al. [6], in their 3D decomposition, apply zero BCs along the entire boundary (hereafter referred to as Tong BCs), such that the irrotational component is normal and the incompressible component parallel to the boundary. Appendix A illustrates the difference between these BC schemes using a 9 value CF potential. In light of these differences, we devised a study to investigate the effect that different BCs have on the decomposition of 2D MVFs with the aim of obtaining a robust and efficient methodology, and a reliable algorithm for application to 2D+time cardiac motion fields. The objectives of this work are: (i) investigate the effect of imposing different sets of BCs, comparing Guo and Tong BCs; and (ii) apply the decomposition to 2D synthetic cardiac MVFs to demonstrate automated extraction of rotational, radial and linear flow. 


\section{Materials}

Three MVFs were created:

1. $\overrightarrow{\xi_{1}}$, formed by superposing a pure rotational (counterclockwise) and radial (sink) field, in turn created by applying differential operators to Gaussian potential fields;

2. $\overrightarrow{\xi_{2}}$, a superposition of $\overrightarrow{\xi_{1}}$ and a linear flow component of $\left(V_{x}, V_{y}\right)=(0,1)$ pixel per frame;

3. $\overrightarrow{\xi_{3}}$, a 2D short axis basal slice through a synthetic cardiac left ventricular MVF produced by the 4D Extended Cardiac-Torso (XCAT) Phantom Version 2.0 [7] using a procedure outlined elsewhere [8].

The 2D scalar Gaussian potentials, used to create $\vec{\xi}_{1}$ and $\overrightarrow{\xi_{2}}$, were defined by $\phi=e^{-\left(1 / 2 \sigma^{2}\right)\left[\left(x-x_{c}\right)^{2}+\left(y-y_{c}\right)^{2}\right]}$ and formed on a $60 \times 60$ pixel grid. In this equation, $\sigma$ is the Gaussian potential standard deviation and $x_{c}$ and $y_{c}$ are the maximum or minimum points of the potential, corresponding to the centre of the radial or rotational component. The centers of the components were set to $(29,31)$ and $(31,29)$ respectively for the radial (sink) and rotational (counterclockwise) fields. Six MVF variations were produced from potential fields with varying smoothness and extent, with $\sigma=2.0,5.0,7.0,9.0,12.0,16.0$.

Image processing software and DHHD were written using Matlab 9.2 (Natwick, Math Works Inc., 2017).

\section{Methodology}

Experiment 1: investigation of boundary conditions. Two versions of the 2D DHHD were implemented, DHHD(i) using Tong BCs and DHHD(ii) using Guo BCs. Experiments were then performed to test the effectiveness of the DHHD algorithms by comparing the rotational, radial and linear motion field components of the input fields $\overrightarrow{\xi_{1}}$ and $\overrightarrow{\xi_{2}}$ with the respective estimated DF, CF and harmonic components resulting from the decomposition. In every case, the input and estimated MVF components were compared using Normalised Root Mean Square Error (NRMSE). The methodology is similar to that described in Sims et al.[8] but with 2D fields.

First the value of $\sigma$ was found which produced the lowest decomposition error using DHHD(i). The field generated using $\sigma=9.0$ provided the lowest NRMSE error $(0.92 \%)$, since the field is as smooth as possible while maintaining very small values at the boundary. This value of $\sigma$ was used to generate radial and rotational MVFs for the remainder of Experiment 1.

The difference between rotational, radial and linear motion field components and their estimated components was investigated (CF, DF and harmonic) when different BCs were applied. Sub-experiments were defined as follows (see Table 1): Experiments $2 \mathrm{a}$ and $2 \mathrm{~b}$ used input fields $\overrightarrow{\xi_{1}}$ and $\overrightarrow{\xi_{2}}$ respectively, being decomposed using algorithm
Table 1: Percent NRMSE for experiments $2 \mathrm{a}-\mathrm{d}$.

\begin{tabular}{ccc}
\hline \hline $\begin{array}{c}\text { Algo- } \\
\text { rithm }\end{array}$ & $\begin{array}{c}\text { \% NRMSE } \\
\text { for } \overrightarrow{\xi_{1}}\end{array}$ & $\begin{array}{c}\text { \% NRMSE for } \\
\overrightarrow{\xi_{2}}\end{array}$ \\
\hline DHHD(i) & $0.92 \%$ & $0.92 \%$ \\
& $($ Exp.2a) & $($ Exp.2b) \\
DHHD(ii) & $0.88 \%$ & $227.79 \%$ \\
& $($ Exp.2c) & (Exp.2d) \\
\hline \hline
\end{tabular}

DHHD(i) (Tong BCs); Experiments 2c and 2d used input fields $\overrightarrow{\xi_{1}}$ and $\overrightarrow{\xi_{2}}$ respectively, being decomposed with algorithm DHHD(ii) (Guo BCs) In all cases, the estimated harmonic (linear) flow, $\vec{F}_{H . e s t}$ was found by calculating the harmonic remainder, $\vec{F}_{H . e s t}=\vec{\xi}-\vec{F}_{C F . e s t}-\vec{F}_{D F \text {.est }}$, as described elsewhere [9].

Experiment 2: decomposition of $\overrightarrow{\xi_{3}}$.

DHHD(i) was applied to the short axis slice through the XCAT MVF and the resulting CF and DF fields presented.

\section{Results}

\section{Experiment 1}

Table 1 shows the results of NRMSE for the subexperiments $2 \mathrm{a}-\mathrm{d}$. 2a, $\mathrm{b}$ and c provide very similar results, with error just under $1 \%$, while sub-experiment $2 \mathrm{~d}$, motion field decomposed by DHHD(ii), produced an error of more than 200\%. This shows that DHHD(i) and (ii) perform similarly in the absence of a harmonic component. When this component is present, DHHD(i) continues to perform well but DHHD(ii) produces very large errors.

An analysis of the harmonic components in the input and output fields are shown in Table 2. Input harmonic components in $\vec{\xi}_{2}$ were $\left(V_{x}, V_{y}\right)=(0,1)$. Mean velocity vector components for the estimated harmonic component in experiment $2 \mathrm{~b}$ were $(0.0003,1.0000)$, close to the input values, while experiment $2 \mathrm{~d}$ produced $(0.0002,-1.0000)$, with the value of $V_{y}$ in the opposite direction to that expected.

Table 2: Estimated harmonic component from decomposition of $\vec{\xi}_{2}$, containing $x$ and $y$ components of velocity, $V_{x}=0$ and $V_{y}=1$. The estimated fields are mean harmonic components from the output of experiments $2 b$ and $2 \mathrm{~d}$.

\begin{tabular}{c|cc}
\hline \hline & $V_{x}$ & $V_{y}$ \\
\hline Input linear component in $\vec{\xi}_{2}:$ & 0 & 1 \\
Estimated linear components: & & \\
DHHD(i) (exp. 2b) & 0.0003 & 1.0000 \\
DHHD(ii) (exp. 2d) & 0.0002 & -1.0000 \\
\hline \hline
\end{tabular}

Figure 1 provides a comparison of the resulting motion 

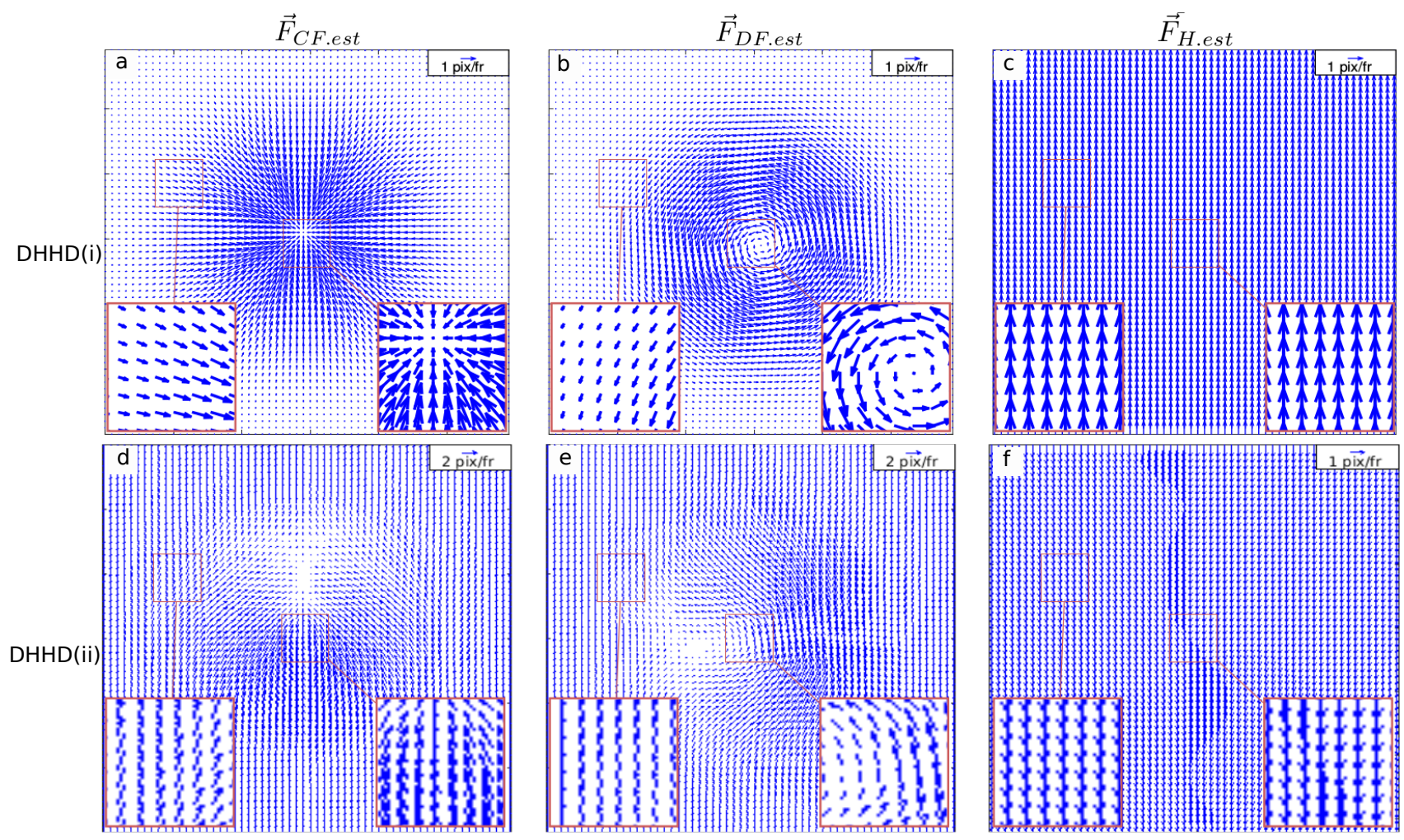

Figure 1: Estimated MVF components resulting from decomposition of $\overrightarrow{\xi_{2}}$ ( sink, counter-clockwise rotation and linear fields superposed). Upper and lower rows result from using the DHHD(i) and DHHD(ii) algorithms respectively (experiments $2 \mathrm{~b}$ and 2d). (a) and (d) are curl-free, $\vec{F}_{C F . e s t}$; (b),(e) divergence-free $\vec{F}_{D F . e s t}$; (c),(f) harmonic $\vec{F}_{H . e s t}$. DHHD(ii) seems not to have separated $\vec{F}_{C F . e s t}$ and $\vec{F}_{D F . e s t}$ from the harmonic input component

field components resulting from the decomposition of $\vec{\xi}_{2}$ using DHHD(i) and DHHD(ii). Subfigures (a), (b), (c) show CF, DF and hamonic components resulting from application of DHHD(i), while (d),(e),(f) result from application of DHHD(ii). DHHD(i) produces the expected result in which the estimated CF field, $\vec{F}_{C F \text {.est }}$, is radial, $\vec{F}_{D F . e s t}$ is rotational and the estimated harmonic field, $\vec{F}_{H . e s t}$ uniform in a positive y direction. However, DHHD(ii) produces MVFs in which $\vec{F}_{C F \text {.est }}$ and $\vec{F}_{D F \text {.est }}$ are superpositions of radial and harmonic input fields, and rotational and harmonic input fields, respectively. The harmonic field points unexpectedly in a negative vertical direction.

\section{Experiment 2}

Figure 2 shows $\mathrm{CF}$ and DF fields for the decomposed XCAT motion field in systole, (frame 4 of a 16 frame sequence), providing clear separation into a CF component, perpendicular to the LV midmyocardial line, and a DF component, largely parallel to this line.

\section{Discussion}

\section{Experiment 1}

As previously mentioned, Figure 1 suggests that
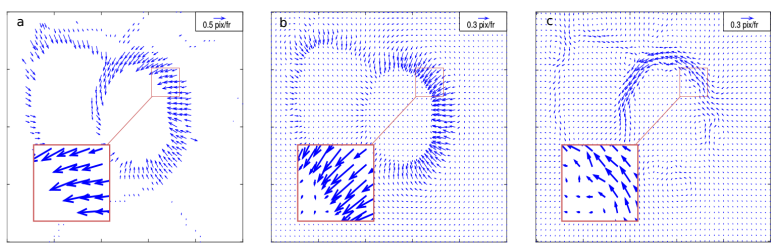

Figure 2: Decomposition of single basal XCAT MVF SAX 2D slice in systole using DHHD(ii). (a) input field, $\vec{\xi}_{3}$; (b) CF field; (c) DF field.

DHHD(ii) produced CF and DF fields with the harmonic component superposed on the radial or rotational field, such that $\vec{F}_{C F . e s t} \approx \vec{F}_{C F}+\vec{F}_{H}$ and $\vec{F}_{D F . e s t} \approx \vec{F}_{D F}+\vec{F}_{H}$. In this case, the estimated harmonic field was found to be $\left(V_{x}, V_{y}\right)=(0.0002,-1.0000)$. Since $\vec{F}_{H \text {.est }}$ is defined as $\vec{\xi}_{2}-\vec{F}_{C F_{\text {eest }}}-\vec{F}_{D F_{\text {e est }}}$, in this case we have a net field of $\vec{\xi}_{2}-\vec{F}_{C F \text {.est }}-\vec{F}_{H}-\vec{F}_{D F \text {.est }}-\vec{F}_{H}$. Since $\overrightarrow{\xi_{2}}=\vec{F}_{C F}+\vec{F}_{C F}+\vec{F}_{H}$, the result is $-\vec{F}_{H}$ plus some small errors between the real and estimated CF and DF fields. This is visualized as the harmonic field pointing in the opposite direction to that expected. 
In finite element methods, the BCs define the problem to be addressed. It seems that Guo BCs produce reliable results in the absence of harmonic flow, whereas Tong BCs produce robust results both with and without harmonic flow. While Guo et al. [9] claim that setting potentials to zero at only one vertex is sufficient for a unique decomposition, they do not claim orthogonality [4]. Further, while they propose that better accuracy in the results can be obtained from further decompositon of the harmonic field, our experiments suggest that inaccuracies are due to the harmonic field being included in $\mathrm{CF}$ and $\mathrm{DF}$ fields rather than CF and DF fields appearing in the harmonic field.

The application of BCs to a single node makes values on the boundary behave in a spontaneous manner, but does not provide sufficient information for the problem.

\section{Experiment 2:}

Applying DHHD(i) to the XCAT MVF, $\vec{\xi}_{3}$, a clear separation of radial and rotational fields was obtained. This MVF has a very low harmonic component, so similar results would be obtained when applying DHHD(ii). In cardiac motion fields obtained from modalities such as $2 \mathrm{D}$ speckle tracking, we expect the harmonic component to be small in the short axis plane.

A qualitative assessment of the $\mathrm{CF}$ and DF fields were found to contain components perpendicular and parallel to the midmyocardial line, respectively. The circumferential component would seem to be useful for automated quantification of LV rotation, from which LV twist can be determined.

\section{Conclusion}

Applying zero BCs to all nodes on the boundary [6] achieves the required decomposition, while applying a zero BC to a single node [9] leads to large errors when a harmonic component is present in the input field. When the DHHD was applied to a $2 \mathrm{D}$ synthetic MVF, $F_{D F}$ was seen to isolate the rotational component. The DHHD is a possible candidate for estimating the rotational component of a cardiac MVF.

\section{Appendix A}

For the curl-free field, $[D]=\left[\begin{array}{ccc}D_{1} & D_{4} & D_{7} \\ D_{2} & D_{5} & D_{8} \\ D_{3} & D_{6} & D_{9}\end{array}\right]$

Guo and Tong BCs, $\overrightarrow{d_{G}}, \overrightarrow{d_{T}}$ (D vectorized) are:

$\overrightarrow{d_{G}}=\left[\begin{array}{lllllllll}0 & D_{2} & D_{3} & D_{4} & D_{5} & D_{6} & D_{7} & D_{8} & D_{9}\end{array}\right]^{T}$

$\overrightarrow{d_{T}}=\left[\begin{array}{lllllllll}0 & 0 & 0 & 0 & D_{5} & 0 & 0 & 0 & 0\end{array}\right]^{T}$

The linear system of equations to find $[\mathrm{D}]$ is $[S] \vec{d}=I \vec{b}$
Write Tong BCs (for example):

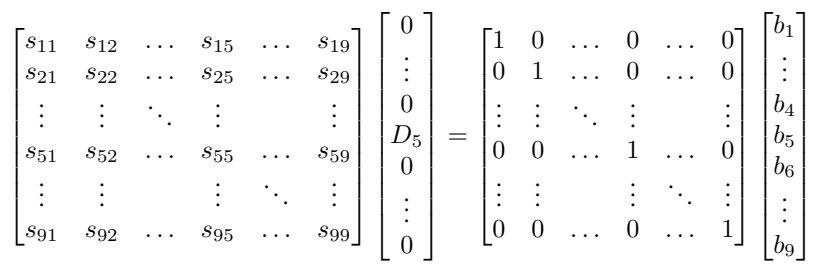

$S$ is a sparse matrix, dependent on the domain triangulation, and $\vec{b}$ depends on MVF and triangulation. For further details, see Sims [10], Guo et al. [9] and Tong et al. [6].

\section{References}

[1] Notomi Y, Lysyansky P, Setser RM, Shiota T, Popović ZB, Martin-Miklovic MG, Weaver JA, Oryszak SJ, Greenberg NL, White RD, Thomas JD. Measurement of ventricular torsion by two-dimensional ultrasound speckle tracking imaging. Journal of the American College of Cardiology 2005;45(12):2034-2041.

[2] Sims JA, Giorgi MC, Oliveira MA, Meneghetti JC, Gutierrez MA. Directional analysis of cardiac motion field from gated fluorodeoxyglucose PET images using the Discrete Helmholtz Hodge Decomposition. Computerized Medical Imaging and Graphics 2018;65:69-78. ISSN 18790771.

[3] Polthier K, Preuss E. Variational approach to vector field decomposition. In de Leeuw W, van Liere R (eds.), Data Visualization 2000, Eurographics. Amsterdam, Holland: Springer Vienna, 2000; 147-155.

[4] Bhatia H, Norgard G, Pascucci V, Bremer PT. The helmholtz-hodge decomposition; a survey. IEEE Transactions on Visualization and Computer Graphics Aug 2013; 19(8):1386-1404. ISSN 1077-2626.

[5] Guo Q, Mandal MK, Li MY. Efficient hodge-helmholtz decomposition of motion fields. Pattern Recogn Lett mar 2005;26(4):493-501.

[6] Tong Y, Lombeyda S, Hirani A, Desbrun M. Discrete multiscale vector field decomposition. ACM Transactions on Graphics 2003;22(3):445-452.

[7] Segars W, Sturgeon G, Mendonca S, Grimes J, Tsui B. 4d XCAT phantom for multimodality imaging research. Medical Physics 2010;37(9):4902-4915.

[8] Sims JA, Macedo MMG, Gutierrez MA. Directional analysis of cardiac motion field based on the discrete helmholtz hodge decomposition. In 2016 Computing in Cardiology Conference (CinC), volume 43. Sept 2016; 493-496.

[9] Guo Q, Mandal MK, Liu G, Kavanagh KM. Cardiac video analysis using hodge-helmholtz field decomposition. Comput Biol Med January 2006;36(1):1-20.

[10] Sims J. Directional Analysis of Cardiac Left Ventricular Motion from PET Images. Ph.D. thesis, Escola politecnica, University of Sao Paulo, Brazil, 2017.

Address for correspondence:

Prof. Dr. John Andrew Sims

Universidade Federal in ABC, UFABC

Alameda da Universidade s/n, Bairro Anchieta,

São Bernardo do Campo, SP, CEP 09606-045

Page 4ohn.sims@ufabc.edu.br 